\title{
Editorial
}

\section{Nano for Biomimetics and Biomaterials}

\author{
Il-Kwon Oh, ${ }^{1}$ Anchal Srivastava, ${ }^{2}$ In-Kyu Park, ${ }^{3}$ and Michael Z. Hu ${ }^{4}$ \\ ${ }^{1}$ Graphene Research Center, School of Mechanical, Aerospace and Systems Engineering, Division of Ocean Systems Engineering, \\ Korea Advanced Institute of Science and Technology (KAIST), 335 Gwahak-ro, Yuseong-gu, Daejeon 305-701, Republic of Korea \\ ${ }^{2}$ Department of Physics, Banaras Hindu University, Varanasi 221005, India \\ ${ }^{3}$ Department of Biomedical Sciences, Chonnam National University, 300 Yongbong-dong, Buk-gu, \\ Gwang-Ju 500-757, Republic of Korea \\ ${ }^{4}$ Oak Ridge National Laboratory, 4500N, A34, MS 6181, Oak Ridge, TN 37831-6181, USA \\ Correspondence should be addressed to Il-Kwon Oh; ikoh@kaist.ac.kr
}

Received 3 April 2014; Accepted 3 April 2014; Published 29 June 2014

Copyright ( 2014 Il-Kwon Oh et al. This is an open access article distributed under the Creative Commons Attribution License, which permits unrestricted use, distribution, and reproduction in any medium, provided the original work is properly cited.

This special issue has been considered as a necessary technology which can overcome the limitations and problems of other modern technologies and lead to a new industrial revolution. The future promise of nanotechnology depends on its high multidisciplinary nature and shared knowledge and information from various fields which can be merged and used to evolve as a revolutionary new technology. Considering the potential impact of nanotechnology on future industry, many countries are investing huge research funds and resources in the field of nanotechnology as one of their top research priorities.

The aim of the special issue is to share the novel knowledge covering the state of the art on biomimetics and nanobiomaterials providing an overview on their potential applications in the industrial, biomedical, and robotic fields. The research topics covered in the special issue include bioinspired materials, devices, structures, and graphenebased materials. The special issue will present current status of the fields of biomimetics and biomaterials. This special issue will be a necessary platform for ongoing studies between researchers from different areas (chemistry, physics, biology, medicine, engineering, robotics, etc.) within biomimetic and biomaterial technologies.

We hope that this special issue reflects the current state of nanotechnology for biomimetics and biomaterials and will be a useful reference for researchers working in this research field.

\section{Acknowledgment}

The guest editors and other colleagues from various institutions offered invaluable support for peer-reviewing and the successful completion of this special issue. We sincerely thank all reviewers and authors of this special issue.

Il-Kwon Oh Anchal Srivastava In-Kyu Park Michael Z. Hu 

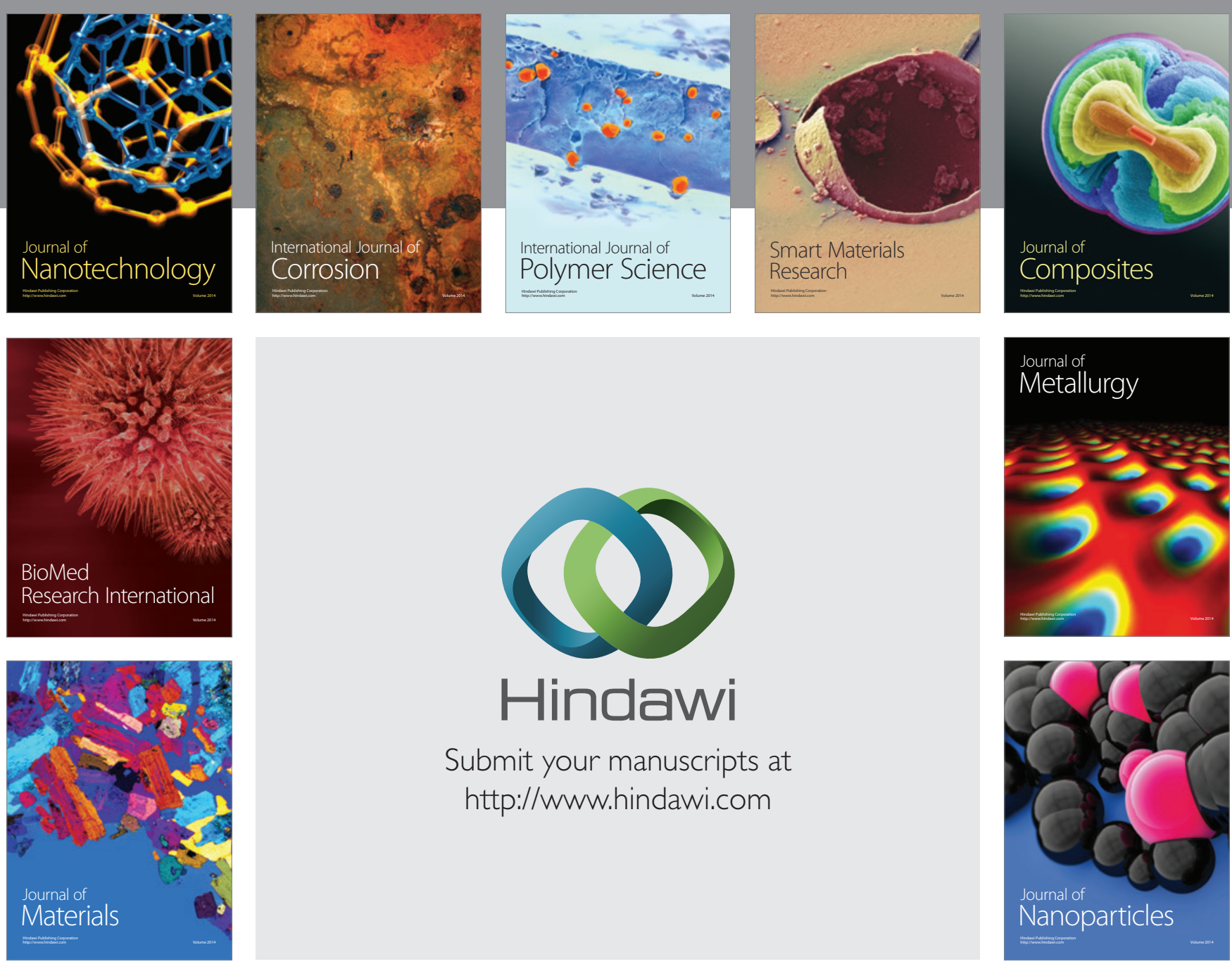

Submit your manuscripts at http://www.hindawi.com
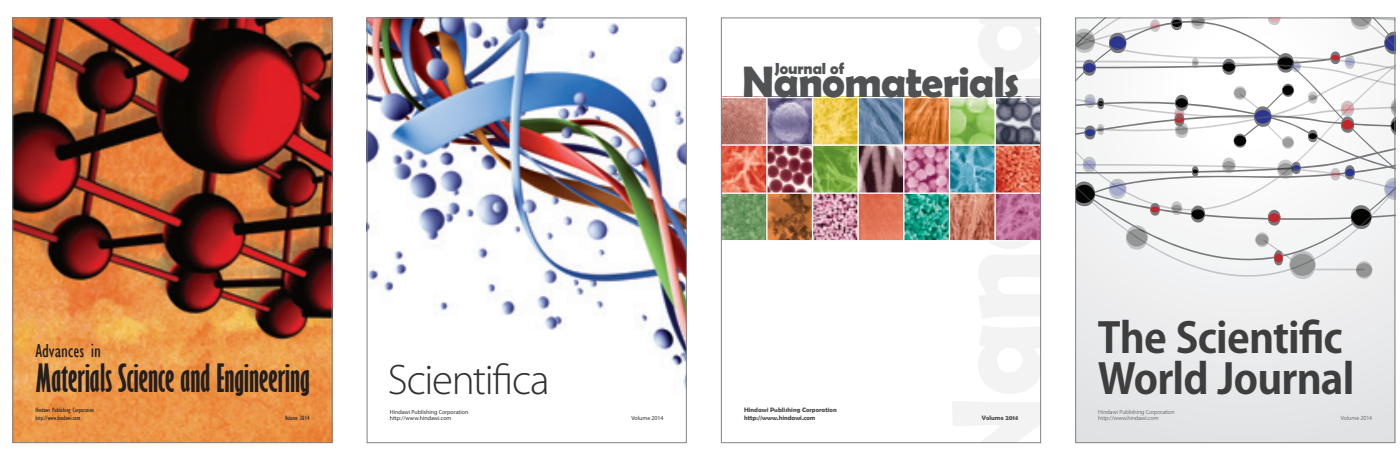

\section{The Scientific World Journal}
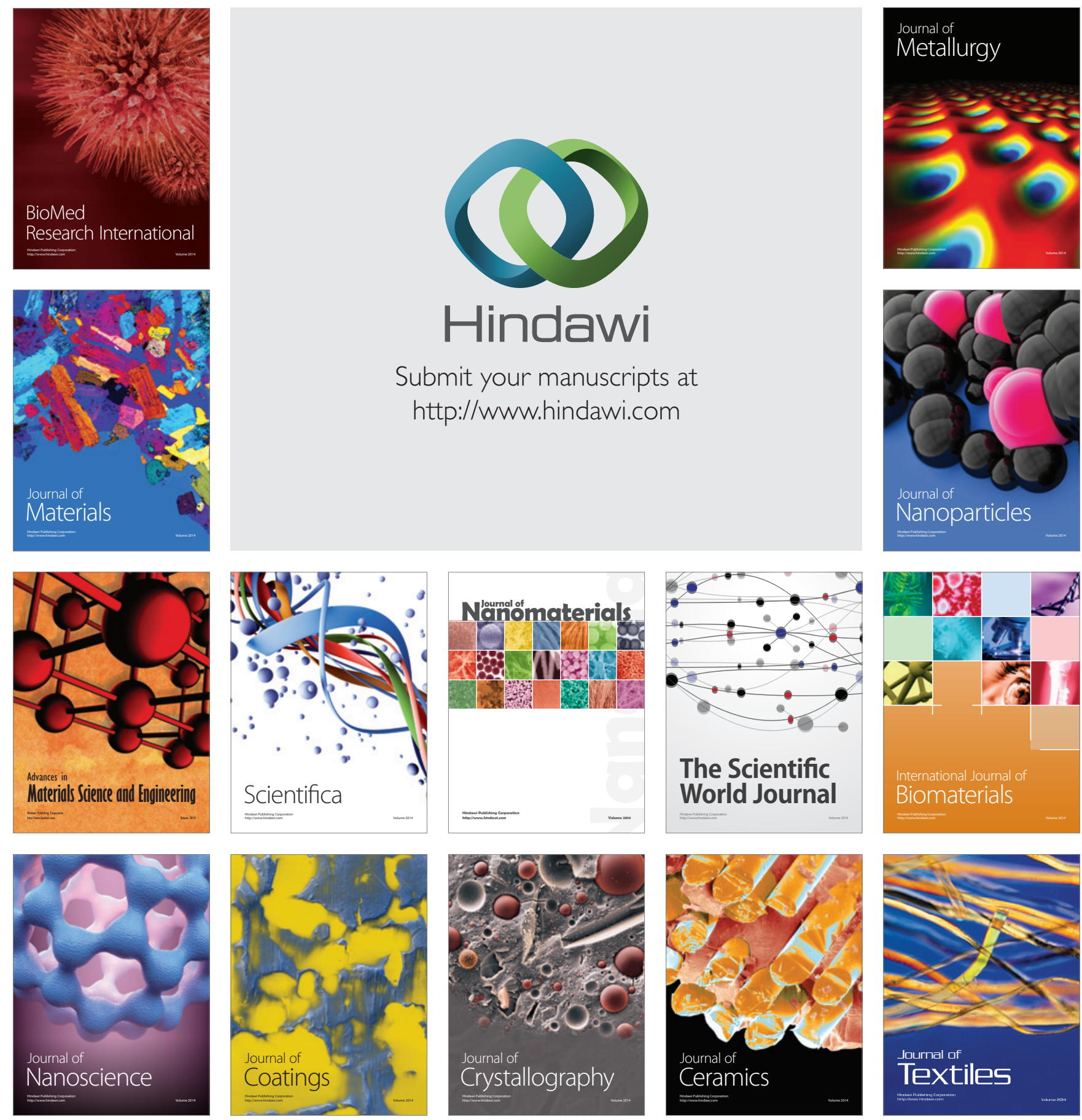